\title{
Vertical Transport of Momentum by the Inertial-Gravity Internal Waves in a Baroclinic Current
}

\author{
A. A. Slepyshev ${ }^{1, *}$, D. I. Vorotnikov ${ }^{2}$ \\ ${ }^{1}$ Marine Hydrophysical Institute, Russian Academy of Sciences, Sevastopol, Russian Federation \\ ${ }^{2}$ M. V. Lomonosov Moscow State University, Moscow, Russian Federation \\ *e-mail: slep55@mail.ru
}

\begin{abstract}
When the internal waves break, they are one of the sources of small-scale turbulence. Small-scale turbulence causes the vertical exchange in the ocean. However, internal waves with regard to the Earth rotation in the presence of vertically inhomogeneous two-dimensional current are able to contribute to the vertical transport. Free inertial-gravity internal waves in a baroclinic current in a boundless basin of a constant depth are considered in the Bussinesq approximation. Boundary value problem of linear approximation for the vertical velocity amplitude of internal waves has complex coefficients when current velocity component, which is transversal to the wave propagation direction, depends on the vertical coordinate (taking into account the rotation of the Earth). Eigenfunction and wave frequency are complex, and it is shown that a weak wave damping takes place. Dispersive relation and wave damping decrement are calculated in the linear approximation. At a fixed wave number damping decrement of the second mode is larger (in the absolute value) than the one of the first mode. The equation for vertical velocity amplitude for real profiles of the Brunt - Vaisala frequency and current velocity are numerically solved according to implicit Adams scheme of the third order of accuracy. The dispersive curves of the first two modes do not reach inertial frequency in the low-frequency area due to the effect of critical layers in which wave frequency of the Doppler shift is equal to the inertial one. Termination of the second mode dispersive curves takes place at higher frequency than the one of the first mode. In the second order of the wave amplitude the Stokes drift speed is determined. It is shown that the Stokes drift speed, which is transversal to the wave propagation direction, differs from zero if the transversal component of current velocity depends on the vertical coordinate. In this case, the Stokes drift speed in the second mode is lower than in the first mode only in the pycnocline, outside the pycnocline their values are comparable in absolute value. The longitudinal component of the Stokes drift velocity of 15-min second mode internal waves observed in the field experiment during the $44^{\text {th }}$ voyage of R/V "Mikhail Lomonosov" on the northwestern shelf of the Black Sea is by an order of magnitude greater than the transversal one. Vertical wave fluxes of the momentum also differ from zero and can be either comparable with the corresponding turbulent fluxes or exceed them.
\end{abstract}

Keywords: internal waves, turbulence, momentum fluxes, Stokes drift, critical layer.

DOI: 10.22449/1573-160X-2017-4-3-15

(C) 2017, A. A. Slepyshev, D. I. Vorotnikov

(C) 2017, Physical Oceanography

Introduction. Vertical exchange plays a key role in a formation of oxygen and hydrogen sulfide concentration fields in the Black Sea. Mixing processes perform the ventilation of waters and maintain a stable functioning of the ecosystem. Usually, vertical exchange in a stratified marine environment is associated with smallscale turbulence which is generated by hydrodynamic instability of currents and breaking of internal waves. The internal waves play an important role in dynamic processes at a sea shelf as energy sources that generate these waves are constantly presented. These sources are the following: atmospheric pressure fluctuations, wind stresses on the sea surface, interaction of currents and tides with inhomogeneities of a bottom topography, instability of currents. 
The effect of small-scale turbulence on the internal waves was considered in a series of works [1-3]. It is shown that internal waves damp when turbulent viscosity and diffusion are taken into account. Vertical wave fluxes of heat, salt and momentum differ from zero at that [4]. In [5, 6] the mentioned effects were considered at the current with vertical shift of speed. It is revealed that without taking into account the rotation of the Earth, critical layers are absent due to the low velocity of current (under $0.15 \mathrm{~m} / \mathrm{s}$ ); its effect is manifested on the magnitude of the wave fluxes. It is of interest to find wave flux of momentum in a vertically-inhomogeneous current for inertial-gravity internal waves (with regard to the Earth rotation). It is noteworthy that the vertical flux of momentum differs from zero in this case, even when turbulent viscosity and diffusion are ignored.

The statement of the problem. Free internal waves in a boundless basin of constant depth are considered taking into account the rotation of the Earth. Two current speed components depend on vertical coordinate. Dispersion relation and vertical distribution of internal wave amplitude are calculated in the linear approximation. The equation for the vertical speed amplitude has complex coefficients, therefore the eigenfunction of internal waves and wave frequency are complex, i. e. a weak wave damping takes place. Vertical wave fluxes of momentum and Stokes drift speed are defined in the second order of amplitude. Hydrodynamics equations in the Bussinesq approximation for wave disturbances have the following form:

$$
\begin{gathered}
\frac{D u}{D t}-f v+w \frac{d U_{0}}{d z}=-\frac{1}{\rho_{0}(0)} \frac{\partial P}{\partial x}, \\
\frac{D v}{D t}+f u+w \frac{d V_{0}}{d z}=-\frac{1}{\rho_{0}(0)} \frac{\partial P}{\partial y}, \\
\frac{D w}{D t}=-\frac{1}{\rho_{0}(0)} \frac{\partial P}{\partial z}-\frac{g \rho}{\rho_{0}(0)}, \\
\frac{D \rho}{D t}=-w \frac{d \rho_{0}}{d z}, \\
\frac{\partial u}{\partial x}+\frac{\partial v}{\partial y}+\frac{\partial w}{\partial z}=0,
\end{gathered}
$$

where $u, v, w$ are two horizontal and vertical components of current wave speed, respectively; $\rho, P$ are wave disturbances of density and pressure; $\rho_{0}(z)$ is a mean density profile; $x, y, z$ are two horizontal and a vertical coordinates, $z$ axis is directed vertically upwards; $f$ is the Coriolis parameter; $U_{0}(z), V_{0}(z)$ are two components of mean current speed; $\frac{D}{D t}$ operator action is revealed by the following formula: $\frac{D}{D t}=\frac{\partial}{\partial t}+\left(u+U_{0}\right) \frac{\partial}{\partial x}+\left(v+V_{0}\right) \frac{\partial}{\partial y}+w \frac{\partial}{\partial z}$. Using the geostrophic relationships [7], we make an estimation of the horizontal scales of mean density variations: 


$$
L_{x}=\rho_{0} / \frac{\partial \rho_{0}}{\partial x}=g /\left(f \max \left|\frac{\partial V_{0}}{\partial z}\right|\right), L_{y}=\rho_{0} / \frac{\partial \rho_{0}}{\partial y}=g /\left(f \max \left|\frac{\partial U_{0}}{\partial z}\right|\right) .
$$

Vertical profiles of current speed components and of their derivatives are represented in Fig. 1. $U_{0}, V_{0}$ maximum values of current speed components vertical gradients make up 0.019 and $8.086 \cdot 10^{-3} \mathrm{c}^{-1}$, respectively. $L_{\mathrm{x}}=1.16 \cdot 10^{7} \mathrm{~m}, L_{\mathrm{y}}=$ $=5 \cdot 10^{6} \mathrm{~m}$ horizontal scales of density variation are much more than the wave length, therefore we neglect the horizontal density variation.
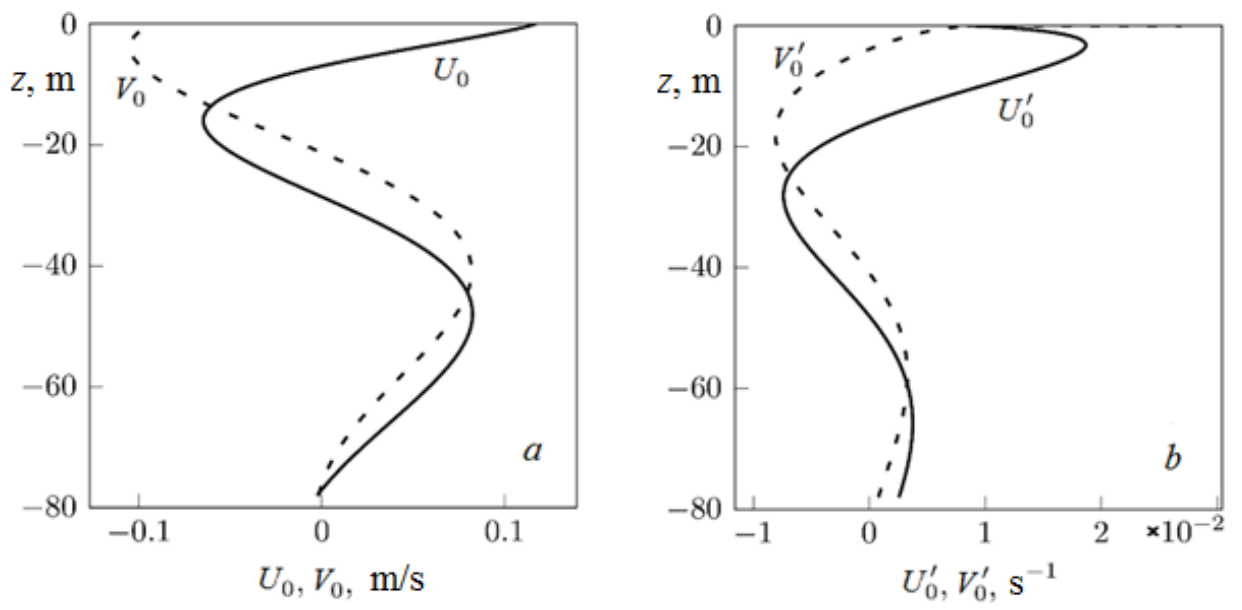

Fig. 1. Vertical profiles of: $V_{0}, U_{0}$ current speed components $-a ; V_{0}^{\prime}, U_{0}^{\prime}$ gradients of current speed components $-b$

Boundary condition on the sea surface $(z=0)$ is a rigid lid condition which filters the internal waves from the surface ones [8]:

$$
w(0)=0 .
$$

Boundary condition on the bottom is an impermeability condition:

$$
w(-H)=0 .
$$

Linear approximation. We seek solutions for the linear approximation in the following form:

$$
\begin{gathered}
u_{1}=u_{10}(z) A \mathrm{e}^{i \theta}+\text { c.c. }, \quad v_{1}=v_{10}(z) A \mathrm{e}^{i \theta}+\text { c.c. }, \quad w_{1}=w_{10}(z) A \mathrm{e}^{i \theta}+\text { c.c. }, \\
P_{1}=P_{10}(z) A \mathrm{e}^{i \theta}+\text { c.c. }, \quad \rho_{1}=\rho_{10}(z) A \mathrm{e}^{i \theta}+\text { c.c. },
\end{gathered}
$$

where c. c. are complex conjugate terms; $A$ is an amplitude multiplier; $\theta$ is a wave phase, $\partial \theta / \partial x=k, \partial \theta / \partial t=-\omega, k$ is a horizontal wave number, $\omega-$ is a wave frequency. It is assumed that the wave propagates along $x$ axis. After substituting (8) into the system (1) - (5), the relation of amplitude functions $u_{10}, v_{10}, P_{10}, \rho_{10}$ to $w_{10}$ function follows: 


$$
\begin{gathered}
u_{10}=\frac{i}{k} \frac{d w_{10}}{d z}, \quad v_{10}=\frac{1}{\Omega}\left(\frac{f}{k} \frac{d w_{10}}{d z}-i w_{10} \frac{d V_{0}}{d z}\right), \\
\frac{P_{10}}{\rho_{0}(0)}=\frac{i}{k}\left[\frac{\Omega}{k} \frac{d w_{10}}{d z}+\frac{d U_{0}}{d z} w_{10}+\frac{f}{\Omega}\left(i \frac{d V_{0}}{d z} w_{10}-\frac{f}{k} \frac{d w_{10}}{d z}\right)\right], \\
\rho_{10}=-\frac{i}{\Omega} w_{10} \frac{d \rho_{0}}{d z}, \quad \Omega=\omega-k U_{0} .
\end{gathered}
$$

$w_{10}$ function satisfies the equation

$$
\begin{aligned}
& \frac{d^{2} w_{10}}{d z^{2}}+k\left[\frac{\text { if } \frac{d V_{0}}{d z}}{\Omega^{2}-f^{2}}-\frac{f^{2} \frac{d U_{0}}{d z}}{\Omega\left(\Omega^{2}-f^{2}\right)}\right] \frac{d w_{10}}{d z}+ \\
& +k w_{10}\left[\frac{k\left(N^{2}-\Omega^{2}\right)+\Omega \frac{d^{2} U_{0}}{d z^{2}}+i f \frac{d^{2} V_{0}}{d z^{2}}}{\Omega^{2}-f^{2}}+\frac{i f k \frac{d U_{0}}{d z} \frac{d V_{0}}{d z}}{\Omega\left(\Omega^{2}-f^{2}\right)}\right]=0,
\end{aligned}
$$

where $N^{2}=-\frac{g}{\rho_{0}(0)} \frac{d \rho_{0}}{d z}$ is a square of the Brunt - Vaisala frequency.

Boundary conditions for $w_{10}$ :

$$
\begin{array}{ll}
\text { at } Z=0 & w_{10}=0, \\
\text { at } Z=-H & w_{10}=0 .
\end{array}
$$

Equation (12) has complex coefficients with a small imaginary part, so we proceed to dimensionless variables (dimensionless physical values are denoted by a prime):

$$
\begin{aligned}
& z=H z^{\prime}, t=t^{\prime} / \omega_{*}, V_{0}=V_{0}^{\prime} V_{0^{*}}, U_{0}=U_{0}^{\prime} V_{0^{*}}, k=k^{\prime} / H, f=f^{\prime} \omega_{*}, \\
& \omega=\omega^{\prime} \omega_{*}, N=N^{\prime} \omega_{*}, \Omega=\Omega^{\prime} \omega_{*},
\end{aligned}
$$

where $H$ is a sea depth; $\omega_{*}$ is a characteristic wave frequency; $V_{0^{*}}$ is a characteristic value of current speed which is transversal to the direction of wave propagation.

Then, the equation (12) takes the following form:

$$
\begin{aligned}
& \frac{d^{2} w_{10}^{\prime}}{d z^{\prime 2}}+k^{\prime}\left[\frac{i \varepsilon f^{\prime} \frac{d V_{0}^{\prime}}{d z}}{\Omega^{\prime 2}-f^{\prime 2}}-\frac{\varepsilon f^{\prime 2} \frac{d U_{0}^{\prime}}{d z}}{\Omega^{\prime}\left(\Omega^{\prime 2}-f^{\prime 2}\right)}\right] \frac{d w_{10}^{\prime}}{d z^{\prime}}+ \\
& +k^{\prime} w_{10}^{\prime}\left[\frac{k^{\prime}\left(N^{\prime 2}-\Omega^{\prime 2}\right)+\varepsilon \Omega^{\prime} \frac{d^{2} U_{0}^{\prime}}{d z^{\prime 2}}+i \varepsilon f^{\prime} \frac{d^{2} V_{0}^{\prime}}{d z^{2}}}{\Omega^{\prime 2}-f^{\prime 2}}+\frac{i \varepsilon^{2} f^{\prime} k^{\prime} \frac{d U_{0}^{\prime}}{d z} \frac{d V_{0}^{\prime}}{d z}}{\Omega^{\prime}\left(\Omega^{\prime 2}-f^{\prime 2}\right)}\right]=0,
\end{aligned}
$$


where $\varepsilon=V_{0^{*}} / H \omega_{*}$ is a small parameter. Imaginary part of coefficients in the equation (16) is of $\varepsilon$ order, therefore the one of $w_{10}$ solution is also proportional to $\varepsilon$, i. e. the solution of the equation (16) is represented as

$$
w_{10}^{\prime}\left(z^{\prime}\right)=w_{0}^{\prime}\left(z^{\prime}\right)+\operatorname{siw}_{1}^{\prime}\left(z^{\prime}\right),
$$

where $w_{0}^{\prime}\left(z^{\prime}\right)$ and $w_{1}^{\prime}\left(z^{\prime}\right)$ are real-valued functions. The frequency is also represented in the form of expansion with respect to $\varepsilon$ parameter:

$$
\omega^{\prime}=\omega_{0}^{\prime}+\varepsilon \sigma_{1}^{\prime}+\ldots,
$$

then $\Omega^{\prime}=\Omega_{0}^{\prime}+\varepsilon \sigma_{1}^{\prime}+\ldots$. After the substitution of (17), (18) into (16), we obtain boundary value problems for $w_{0}^{\prime}\left(z^{\prime}\right)$ and $w_{1}^{\prime}\left(z^{\prime}\right) . w_{0}^{\prime}\left(z^{\prime}\right)$ function satisfies the following equation (within the to $\sim \varepsilon$ terms):

$$
\begin{gathered}
\frac{d^{2} w_{0}^{\prime}}{d z^{\prime 2}}-\varepsilon k^{\prime} \frac{d w_{0}^{\prime}}{d z^{\prime}} \frac{d U_{0}}{d z^{\prime}} \frac{f^{\prime 2}}{\Omega_{0}^{\prime}\left(\Omega_{0}^{\prime 2}-f^{\prime 2}\right)}+ \\
+\frac{k^{\prime} w_{0}^{\prime}}{\left(\Omega_{0}^{\prime 2}-f^{\prime 2}\right)}\left[k^{\prime}\left(N^{\prime 2}-\Omega_{0}^{\prime 2}\right)+\varepsilon \Omega_{0}^{\prime} \frac{d^{2} U_{0}^{\prime}}{d z^{\prime 2}}\right]=0 .
\end{gathered}
$$

Boundary conditions for $w_{0}^{\prime}$

$$
w_{0}^{\prime}(0)=0, \quad w_{0}^{\prime}(-1)=0 .
$$

$w_{1}\left(z^{\prime}\right)$ satisfies the equation (with the accuracy to $\sim \varepsilon$ terms)

$$
\begin{gathered}
\frac{d^{2} w_{1}^{\prime}}{d z^{\prime 2}}-\varepsilon k^{\prime} \frac{d w_{1}^{\prime}}{d z^{\prime}} \frac{d U_{0}^{\prime}}{d z^{\prime}} \frac{f^{\prime 2}}{\Omega_{0}^{\prime}\left(\Omega_{0}^{\prime 2}-f^{\prime 2}\right)}+ \\
+\frac{k^{\prime} w_{1}^{\prime}}{\left(\Omega_{0}^{\prime 2}-f^{\prime 2}\right)}\left[k^{\prime}\left(N^{\prime 2}-\Omega_{0}^{\prime 2}\right)+\delta \Omega_{0}^{\prime} \frac{d^{2} U_{0}^{\prime}}{d z^{\prime 2}}\right]=F^{\prime}\left(z^{\prime}\right),
\end{gathered}
$$

where

$$
\begin{aligned}
& F^{\prime}\left(z^{\prime}\right)=-k^{\prime} \frac{d w_{0}^{\prime}}{d z^{\prime}} \frac{d V_{0}^{\prime}}{d z^{\prime}} \frac{f^{\prime}}{\left(\Omega_{0}^{\prime 2}-f^{\prime 2}\right)}+i k^{\prime} \frac{d w_{0}^{\prime}}{d z^{\prime}} \frac{d U_{0}^{\prime}}{d z^{\prime}} \frac{\sigma_{1}^{\prime} f^{\prime 2}\left(3 \Omega_{0}^{\prime 2}-f^{\prime 2}\right)}{\Omega_{0}^{\prime 2}\left(\Omega_{0}^{\prime 2}-f^{\prime 2}\right)^{2}}- \\
& -\frac{k^{\prime} w_{0}^{\prime}}{\left(\Omega_{0}^{\prime 2}-f^{\prime 2}\right)}\left[k^{\prime} \frac{2 i \Omega_{0}^{\prime} \sigma_{1}^{\prime}\left(N^{\prime 2}-f^{\prime 2}\right)}{\left(\Omega_{0}^{\prime 2}-f^{\prime 2}\right)}+\varepsilon \frac{d^{2} U_{0}^{\prime}}{d z^{\prime 2}} \frac{i \sigma_{1}^{\prime}\left(\Omega_{0}^{\prime 2}+f^{\prime 2}\right)}{\left(\Omega_{0}^{\prime 2}-f^{\prime 2}\right)}\right]- \\
& -\frac{k^{\prime} w_{0}^{\prime}}{\left(\Omega_{0}^{\prime 2}-f^{\prime 2}\right)}\left(f^{\prime} \frac{d^{2} V_{0}^{\prime}}{d z^{\prime 2}}+\varepsilon \frac{f^{\prime} k^{\prime}}{\Omega_{0}^{\prime}} \frac{d U_{0}^{\prime}}{d z^{\prime}} \frac{d V_{0}^{\prime}}{d z^{\prime}}\right) .
\end{aligned}
$$

Boundary conditions for $w_{1}^{\prime}$ function

$$
w_{1}^{\prime}(0)=0, \quad w_{1}^{\prime}(-1)=0 .
$$

PHYSICAL OCEANOGRAPHY ISS. 4 (2017) 
After the transition to dimensional variables, equation (19) takes the form

$$
\begin{aligned}
& \frac{d^{2} w_{0}}{d z^{2}}-k \frac{d w_{0}}{d z} \frac{d U_{0}}{d z} \frac{f^{2}}{\Omega_{0}\left(\Omega_{0}^{2}-f^{2}\right)}+ \\
& +\frac{k w_{0}}{\left(\Omega_{0}^{2}-f^{2}\right)}\left[k\left(N^{2}-\Omega_{0}^{2}\right)+\Omega_{0} \frac{d^{2} U_{0}}{d z^{2}}\right]=0,
\end{aligned}
$$

where $\Omega_{0}=\omega_{0}-k U_{0}$ is a wave frequency with the Doppler shift. The equation (23) should be supplemented by boundary conditions

$$
w_{0}(0)=0, \quad w_{0}(-H)=0 .
$$

In the absence of $\left(U_{0}=0\right)$ current boundary problem (23), (24) at $k$ fixed wave number has a discrete spectrum of frequencies lying in $f<\omega_{0}<\max (N)$ interval. Each $k$ value corresponds to a certain frequency value for the given mode. At $U_{0}(z) \neq 0$ the discrete spectrum can be absent [9], which is due to a presence of singularities in the equation (23) at $\Omega_{0}=0$ and $\Omega_{0}= \pm f$ (hydrodynamically stable currents are considered). In this case there is a critical layer [10] where $\Omega_{0}=0$ (phase velocity of the wave is equal to the one of current at that). The fact that the rotation of the Earth is taken into account leads to critical layer shift to the layer where $\Omega_{0}=f$ [11]. The effect of this critical layer on the dispersion curves is illustrated by the calculations given below. Let $a(z)=-\frac{f^{2} k}{\Omega_{0}\left(\Omega_{0}^{2}-f^{2}\right)} \frac{d U_{0}}{d z}$, $b(z)=\frac{k}{\left(\Omega_{0}^{2}-f^{2}\right)}\left[k\left(N^{2}-\Omega_{0}^{2}\right)+\Omega_{0} \frac{d^{2} U_{0}}{d z^{2}}\right]$, then the equation (23) can be written as

$$
\frac{d^{2} w_{0}}{d z^{2}}+a(z) \frac{d w_{0}}{d z}+b(z) w_{0}=0
$$

The equation (25) is reduced to a self-adjoint form after multiplying the both parts by $p(z)=\exp \left(\int a(z) d z\right)$ :

$$
\frac{d}{d z}\left(p(z) \frac{d w_{0}}{d z}\right)-q(z) w_{0}=0
$$

here $q(z)=-b(z) p(z)$.

After the transition to dimensional variables, the equation (21) is transformed to the following form:

where

$$
\frac{d^{2} w_{1}}{d z^{2}}+a(z) \frac{d w_{1}}{d z}+b(z) w_{1}=F(z),
$$




$$
\begin{aligned}
& F(z)=-k \frac{d w_{0}}{d z} \frac{d V_{0}}{d z} \frac{f}{\left(\Omega_{0}^{2}-f^{2}\right)}+i k \frac{d w_{0}}{d z} \frac{d U_{0}}{d z} \frac{\sigma_{1} f^{2}\left(3 \Omega_{0}^{2}-f^{2}\right)}{\Omega_{0}^{2}\left(\Omega_{0}^{2}-f^{2}\right)^{2}}- \\
& -\frac{k w_{0}}{\left(\Omega_{0}^{2}-f^{2}\right)}\left[k \frac{2 i \Omega_{0} \sigma_{1}\left(N^{2}-f^{2}\right)}{\left(\Omega_{0}^{2}-f^{2}\right)}+i \frac{d^{2} U_{0}}{d z^{2}} \frac{\sigma_{1}\left(\Omega_{0}^{2}+f^{2}\right)}{\left(\Omega_{0}^{2}-f^{2}\right)}\right]- \\
& -\frac{k w_{0}}{\left(\Omega_{0}^{2}-f^{2}\right)}\left(f \frac{d^{2} V_{0}}{d z^{2}}+\frac{f k}{\Omega_{0}} \frac{d U_{0}}{d z} \frac{d V_{0}}{d z}\right) .
\end{aligned}
$$

Boundary conditions for $w_{1}$ function

$$
w_{1}(0)=0, w_{1}(-H)=0 .
$$

The left part of the inhomogeneous linear differential equation (27) is reduced to a self-adjoint form after multiplying the both parts by $p(z)$ function:

$$
\frac{d}{d z}\left(p(z) \frac{d w_{1}}{d z}\right)-q(z) w_{1}=F_{1}(z),
$$

where $F_{1}(z)=p(z) F(z)$.

The solvability condition for the boundary value problem (28), (29) has the following form [12]:

$$
\int_{-H}^{0} F_{1} w_{0} d z=0
$$

from here the expression for $\sigma_{1}$ is found

$$
\sigma_{1}=\frac{q}{r}
$$

where

$$
\begin{aligned}
& q=i f k \int_{-H}^{0} \frac{p w_{0}}{\left(\Omega_{0}^{2}-f^{2}\right)}\left(\frac{d}{d z}\left(w_{0} \frac{d V_{0}}{d z}\right)+w_{0} \frac{k}{\Omega_{0}} \frac{d U_{0}}{d z} \frac{d V_{0}}{d z}\right) d z, \\
& r=\int_{-H}^{0} \frac{p k w_{0}}{\left(\Omega_{0}^{2}-f^{2}\right)^{2}}\left[w_{0}\left(2 k \Omega_{0}\left(N^{2}-f^{2}\right)+\frac{d^{2} U_{0}}{d z^{2}}\left(\Omega_{0}^{2}+f^{2}\right)\right)\right] d z- \\
& -\int_{-H}^{0} \frac{f^{2} p k w_{0}}{\left(\Omega_{0}^{2}-f^{2}\right)^{2}} \frac{d w_{0}}{d z} \frac{d U_{0}}{d z} \frac{\left(3 \Omega_{0}^{2}-f^{2}\right)}{\Omega_{0}^{2}} d z .
\end{aligned}
$$

$\sigma_{1}$ value is purely imaginary, i. e. $\delta \omega=\sigma_{1} / i$ is a decrement of wave damping.

Nonlinear effects. The speed of the Stokes drift of fluid particles is determined by the formula [13]

$$
\mathbf{u}_{\mathrm{s}}=\overline{\int_{0}^{t}(\mathbf{u} d \tau \nabla) \mathbf{u},}
$$

PHYSICAL OCEANOGRAPHY ISS. 4 (2017) 
where $\mathbf{u}$ is a field of the Euler wave velocities; the bar above the formula denotes the averaging over the wave period. The Stokes drift speed horizontal component directed along the wave vector has the following form (with the accuracy to the terms quadratic by the wave amplitude):

$$
u_{\mathrm{s}}=\frac{A_{1} A_{1}^{*}}{k}\left[\frac{1}{\omega} \frac{d}{d z}\left(w_{10} \frac{d w_{10}^{*}}{d z}\right)+\text { c.c. }\right] \text {, }
$$

where $A_{1}=A \exp (\delta \omega \cdot t)$.

The horizontal component of the Stokes drift speed, which is transverse to the wave propagation direction, is determined by the formula

$$
\begin{gathered}
v_{\mathrm{s}}=A_{1} A_{1}^{*}\left[\frac{1}{\omega \Omega^{*} k} \frac{d w_{10}}{d z}\left(\text { if } \frac{d w_{10}^{*}}{d z}-k \frac{d V_{0}}{d z} w_{10}^{*}\right)\right]-A_{1} A_{1}^{*} \frac{w_{10}^{*}}{\omega^{*} \Omega^{2}} \times \\
\times\left[\Omega\left(\frac{d w_{10}}{d z} \frac{d V_{0}}{d z}+\frac{\text { if }}{k} \frac{d^{2} w_{10}}{d z^{2}}+w_{10} \frac{d^{2} V_{0}}{d z^{2}}\right)+k \frac{d U_{0}}{d z}\left(w_{10} \frac{d V_{0}}{d z}+\frac{\text { if }}{k} \frac{d w_{10}}{d z}\right)\right]+\text { c.c. }
\end{gathered}
$$

In the presence of an average current (its $V_{0}$ velocity component, transversal to the wave propagation direction, depends on vertical coordinate), $v_{\mathrm{s}}$ value differs from zero.

Let us find the vertical wave fluxes of $\overline{u w}, \overline{v w}$ momentum taking into account the expansions (17), (18):

$$
\begin{gathered}
\overline{u w} /\left|A_{1}^{2}\right|=\frac{i}{k}\left(w_{10}^{*} \frac{d w_{10}}{d z}-w_{10} \frac{d w_{10}^{*}}{d z}\right), \\
\overline{v w} /\left|A_{1}^{2}\right|=\frac{i w_{10} w_{10}^{*}}{\Omega \Omega^{*}}\left(\Omega-\Omega^{*}\right) \frac{d V_{0}}{d z}+\frac{f k}{\Omega \Omega^{*}}\left(\Omega^{*} w_{10}^{*} \frac{d w_{10}}{d z}+\Omega w_{10} \frac{d w_{10}^{*}}{d z}\right) .
\end{gathered}
$$

$\overline{v w}$ vertical wave momentum flux differs from zero also in the absence of current. $\overline{u w}$ momentum flux differs from zero only in the presence of mean current whose transversal to the wave propagation direction $V_{0}$ velocity component depends on the vertical coordinate.

The results of calculations. To determine the vertical wave fluxes of momentum we use the results of the third stage of $44^{\text {th }}$ voyage of $\mathrm{R} / \mathrm{V}$ "Mikhail Lomonosov" (the Black Sea northwestern shelf). According to the data of gradientdistribution temperature sensors (GRAD instruments) the time course of vertical displacements of the temperature isolines is constructed (Fig. 2) [14]. The instruments were placed one above the other and crossed the following layers: 5-15 m (the first instrument), 15-25 m (the second one), 25-35 m (the third one), 35-60 m (the fourth one). It can be clearly seen that strong 15-min oscillations in 25-60 m depth interval are in antiphase with oscillations in 15-25 m interval. This fact indi- 
cates the presence of the second mode internal waves. The maximum amplitude of these waves made up $0.5 \mathrm{~m}$ and this allowed us to find $A_{1}$ normalizing factor. Indeed, the vertical velocity is linked with $\zeta$ vertical displacement by $\frac{d \zeta}{d t}=w$ relation. Hence $\zeta$ and an expression for $A_{1}$ are found:

$$
\zeta=\frac{i w_{0}}{\Omega_{0}} A_{1} \exp \left(i k x-i \omega_{0} t\right)+\text { c.c., } \quad A_{1}=\frac{\max \zeta}{2 \max \left|w_{0} / \Omega_{0}\right|} .
$$

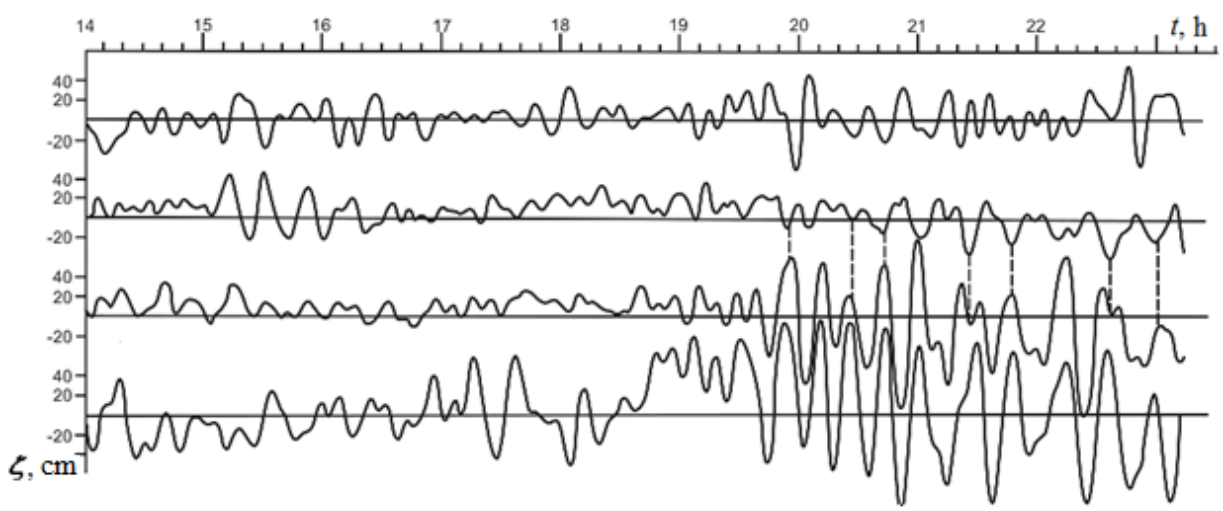

Fig. 2. Temporal course of vertical displacements of temperature isolines

It follows from (36) that the maximum $\zeta$ corresponds to the maximum of $w_{0} / \Omega_{0}$ function, the maximum by modulus value of this relation (according to the calculation data) is reached precisely in the point of $w_{0}$ function maximum. Eigenfunction of the second mode internal waves has its maximum at $50 \mathrm{~m}$ depth (Fig. 3, a), i. e. it corresponds to the maximum elevations according to the experiment data (Fig. 2). The boundary value problem (23), (24) according to $w_{0}$ definition is solved numerically by the implicit Adams scheme of the third order of accuracy. Vertical profile of the Brunt - Vaisala frequency is shown in Fig. 3, $b$. At the fixed wave frequency $k$ wave number is determined using the shooting method (because of necessity of satisfying the boundary conditions (24)). Dispersive curves of the first two modes are represented in Fig. 4, $a$. It is noteworthy that in lowfrequency area dispersive curves do not reach the inertial frequency, moreover, the minimum frequency value of the second mode is higher than of the first one. This is due to the singularity in the equation (23) where the wave frequency with the Doppler shift is equal to the inertial one. We determine the funcntion $w_{1}$ by solving the inhomogeneous boundary value problem (28), (29). From the solvability condition (30) of this boundary value problem, we find the complex correction to the wave frequency, i. e. $\delta \omega$ wave damping decrement. 15-min internal waves of the 
second mode have $\delta \omega=-1.12 \cdot 10^{-5} \mathrm{rad} / \mathrm{s}$. The boundary value problem (28), (29) is solved numerically according to the implicit Adams scheme of the third order of accuracy. The only solution, orthogonal to $w_{0}$ one of the corresponding boundary value problem (23), (24), is found. Damping decrement dependence on the wave number is shown in Fig. 4, $b$. In the low-frequency area this dependence behaves differently for the first and the second mode. This is due to the termination of dispersive curves in the low-frequency area; moreover, the termination of the second mode takes place at higher frequency than the one of the first mode. With the fixed wave number damping decrement of the second mode is greater (by the absolute magnitude) than the one of the first mode.
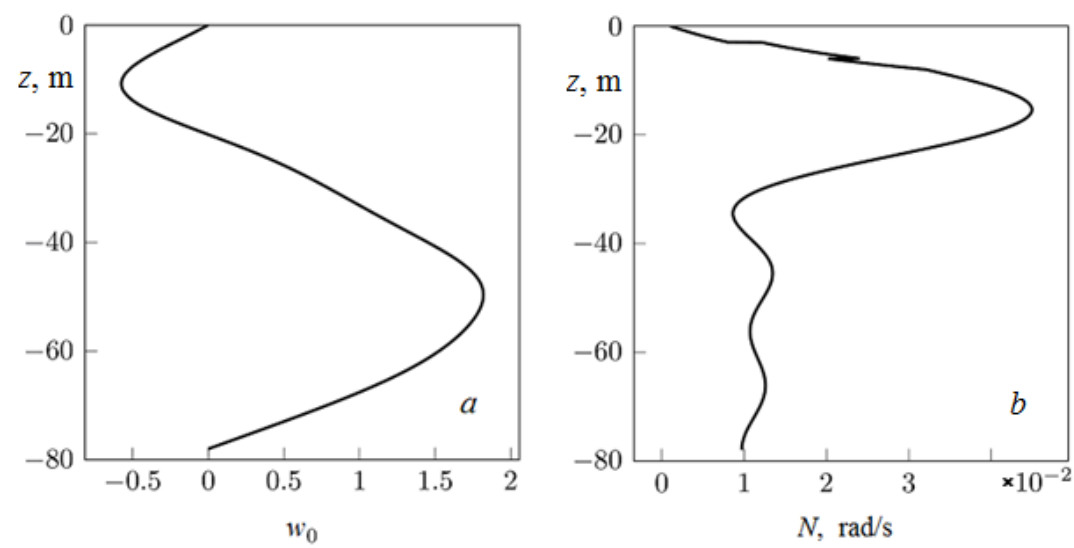

Fig. 3. Eigenfunction of 15-min internal waves of the second mode $-a$ and vertical profile of the Brunt - Vaisala frequency $-b$
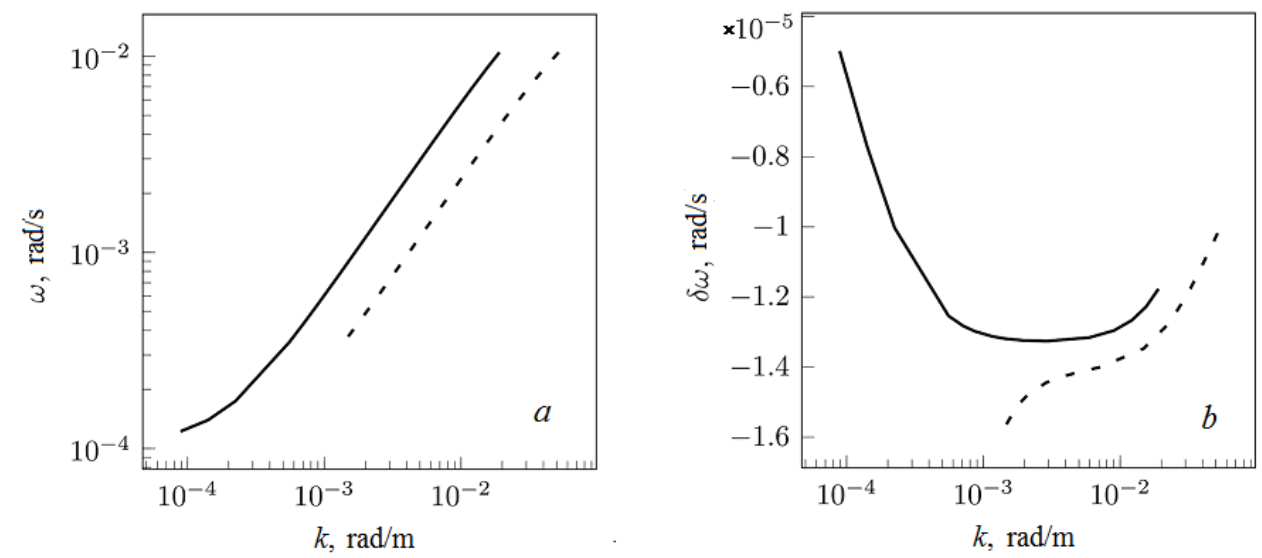

Fig. 4. Dispersive curves of the first and the second modes $-a$; the dependence of wave damping decrement on the wave number $-b$. The first mode is depicted by the solid lines, the second one - by dashed lines

Vertical profiles of the Stokes drift speed horizontal component normalized to the square of the maximum wave amplitude for the first and the second modes of 
15-min internal waves are shown in Fig. 5. The profiles of the Stokes drift speed (33) which is transversal to wave propagation direction is given in Fig. 6. The Stokes drift speed transverse component is by an order smaller than the longitudinal one. The Stokes drift speed of the second mode is lower than the one of the first mode only in a pycnocline at that.
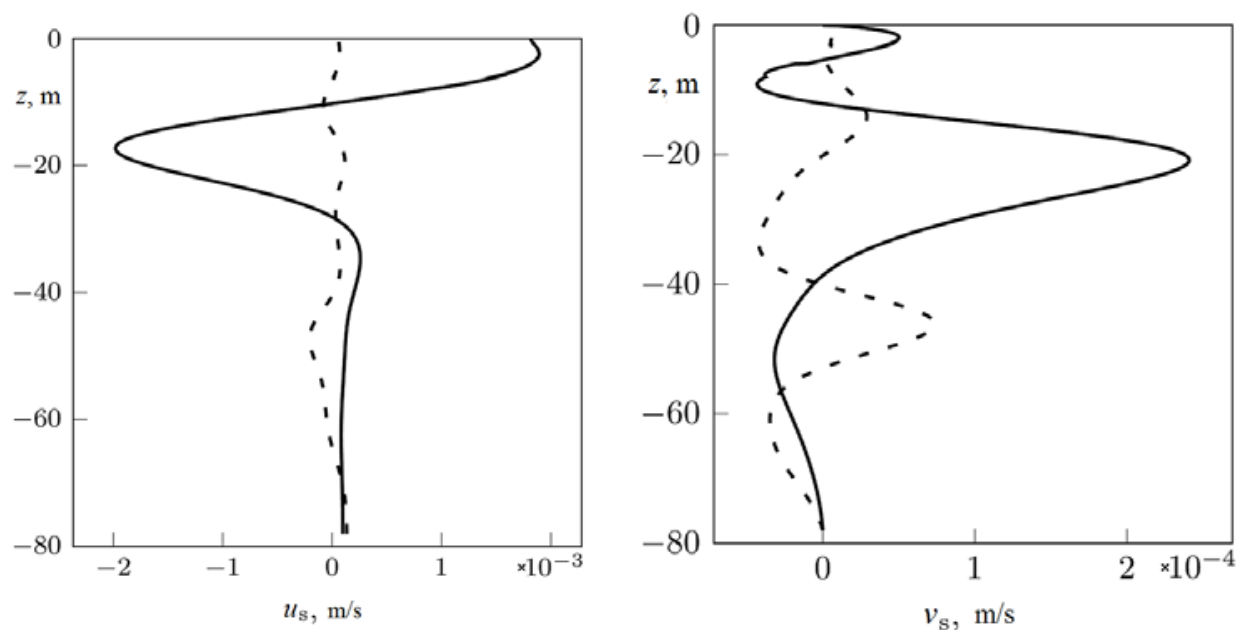

Fig. 5. The Stokes drift speed for the first (solid line) and the second (dashed line) modes Fig. 6. The Stokes drift speed horizontal component (which is transversal to the wave propagation direction) for the first (solid line) and the second (dashed line) modes
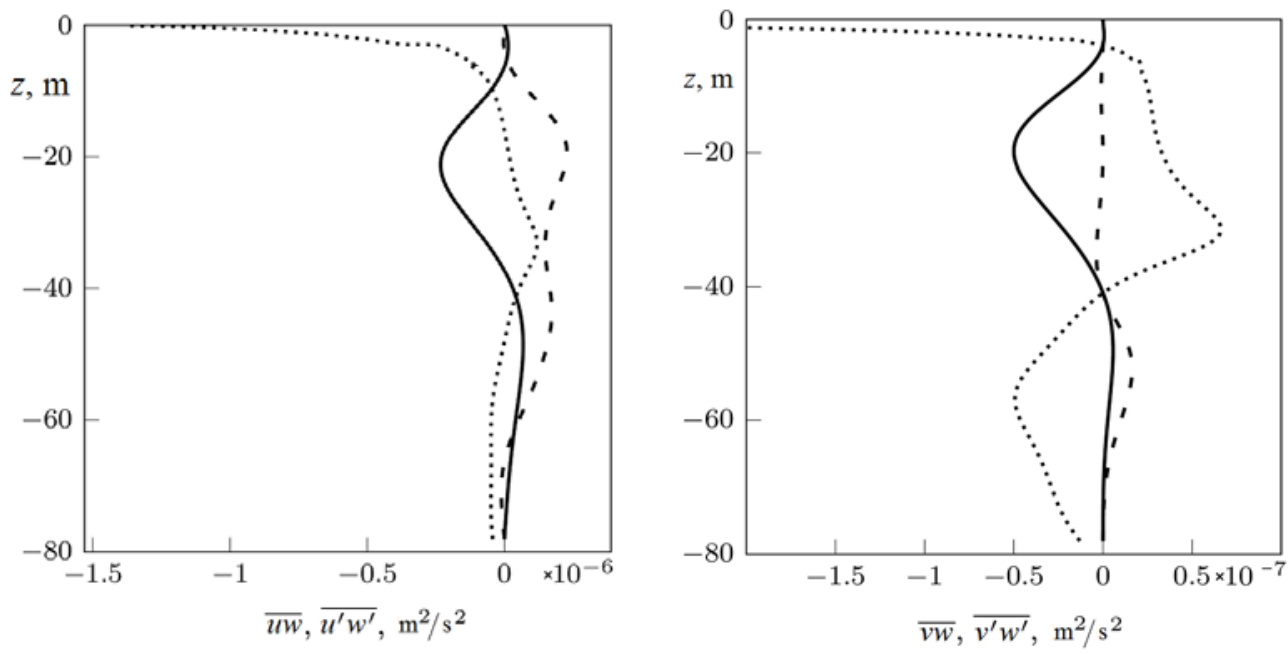

Fig. 7. Profiles of vertical momentum fluxes (solid line - the first mode, dashed line - the second mode, dotted line - turbulent flux)

Fig. 8. Profiles of wave $\overline{v w}$ and turbulent $\overline{v^{\prime} w^{\prime}}$ vertical fluxes of momentum (for notations see Fig. 7)

Vertical profiles of wave $\overline{u w}$ and turbulent $\overline{u^{\prime} w^{\prime}}$ fluxes of momentum are given in Fig.7. Turbulent flux of momentum was determined by $\overline{u^{\prime} w^{\prime}}=-K_{z} \frac{d U_{0}}{d z}$, the PHYSICAL OCEANOGRAPHY ISS. 4 (2017) 
coefficient of vertical turbulent exchange - by $K_{z} \cong 0,93 \cdot 10^{-4} N_{c}^{-1} \mathrm{~m}^{2} / \mathrm{s}\left(N_{c}\right.$, cycle/h is the Brunt - Vaisala frequency) [15]. Wave flux was normalized to the square of wave amplitude. In the upper 10-meter layer turbulent flux exceeds the wave one, in deeper layers these fluxes are comparable by the magnitude. Moreover, in the pycnocline wave flux dominates. The profiles of vertical momentum fluxes $\overline{v w}$ and $\overline{v^{\prime} w^{\prime}}=-K_{z} \frac{d V_{0}}{d z}$ are represented in Fig. 8. In the upper 40-meter layer the flux of the second mode is smaller than the one of the first mode. Turbulent flux prevails on the wave one everywhere, except for the pycnocline where it is comparable in value with the first mode flux.

\section{Conclusions.}

1. Vertical wave fluxes of momentum of inertial-gravity internal waves differ from zero in a baroclinic flux and can be compared with turbulent fluxes (or exceed them).

2. Wave flux $\overline{u w}$ differs from zero only in the presence of current, whose velocity component (which is transversal to the wave propagation direction) depends on the vertical coordinate. In this case the Stokes drift velocity component, which is transversal to the wave propagation direction, differs from zero and is smaller than a longitudinal one by an order of magnitude.

3. Dispersive curves of internal waves of two first modes terminate in a lowfrequency area which is due to the critical layer effect where wave frequency with the Doppler shift is equal to the inertial one. Termination of the second mode occurs at higher frequency that the one of the first mode.

Acknowledgements. The work was carried out within the framework the state order No. 0827-2014-0010 “Complex Interdisciplinary Studies of Oceanographic Processes Determining the Functioning and Evolution of the Black and Azov Sea Ecosystem on the Basis of Modern Methods of Control of the Marine Environment and Grid-technology".

\section{REFERENCES}

1. LeBlond, P.H. and Mysak, L., 1978. Waves in the Ocean. Amsterdam: Elsevier Scientific Publishing Company, 602 p. Available at: https://www.elsevier.com/books/waves-in-theocean/leblond/978-0-444-41926-2 [Accessed 20 June 2017].

2. LeBlond, P.H., 1966. On Damping of Internal Gravity Waves in a Continuously Stratified Ocean. J. Fluid Mech., [e-journal] 25(1), pp. 121-142. doi:10.1017/S0022112066000089

3. Ostrovskiy, L.A. and Soustova, I.A., 1979. The Upper Mixing Layer as a Sink of Internal Wave Energy. Okeanologya, 19(6), pp. 973-981.

4. Slepyshev, A.A., 1997. Transport Processes Induced by Weakly Nonlinear Internal Waves in the Presence of Turbulence. Izvestiya, Atmospheric and Oceanic Physics, 33(4), pp. 494-505.

5. Nosova, A.V. and Slepyshev, A.A., 2015. Vertical Fluxes Induced by Weakly Nonlinear Internal Waves on a Shelf. Fluid Dynamics, [e-journal] 50(1), pp. 12-21. doi:10.1134/S0015462815010020

6. Slepyshev, A.A., 2016. Vertical Momentum Transfer by Internal Waves when Eddy Viscosity and Diffusion are Taken into Account. Izvestiya, Atmospheric and Oceanic Physics, [e-journal] 52(3), pp. 301-308. doi:10.1134/S0001433816030117 
7. Kamenkovich, V.M., 1973. Osnovy Dynamiki Okeana [Basics Ocean Dynamics]. Leningrad: Gidrometeoizdat, 128 p. (in Russian).

8. Miropol'skiy, Yu.Z., 1981. Dynamika Vnutrennikh Gravitatsionnikh Voln v Okeane [Dynamics of Internal Gravity Waves in the Ocean]. Leningrad: Gidrometeoizdat, 302 p. (in Russian).

9. Banks, W.H.H., Drazin, P.G. and Zaturska, M.B., 1976. On the Normal Modes of Parallel Flow of Inviscid Stratified Fluid. J. Fluid Mech., [e-journal] 75(1), pp. 149-171. doi:10.1017/S0022112076000153

10. Booker, J.B. and Brethertone, F.P., 1967. The Critical Layer for Internal Gravity Waves in a Shear Flow. J. Fluid Mech., [e-journal] 27(3), pp. 513-539. doi:10.1017/S0022112067000515

11. Jones, W.L., 1967. Propagation of Internal Waves in Fluids with Shear Flow and Rotation. $J$. Fluid Mech., [e-journal] 30(3), pp. 513-539. doi:10.1017/S0022112067001521

12. Kamke, E., 1959. Differentialgleichungen. Lösungsmethoden und Lösungen. Leipzig: Akademische Verlagsgesellschaft, 244 p.

13. Longuet-Higgins, M.S., 1969. On the Transport of Mass by Time-Varying Ocean Currents. Deep-Sea Res., 16(5), pp. 431-447.

14. MHI AN USSR, 1985. Otchet o Rabotakh v 44 Reise (3 Etap) NIS "Mickhail Lomonosov" 7 Avgusta - 15 Sentyabrya 1985 [Report on Activities of the 44-th Route (3rd Stage) by the Mikhail Lomonosov Research Vessel on August 7-September 15, 1985]. Sevastopol: MHI AS USSR. Vol. 1, 135 p. (in Russian).

15. Ivanov, V.A, Samodurov, A.S., Chukharev, A.M. and Nosova, A.V., 2008. Intencifikatsiya Vertikalnogo Turbulentnogo Obmena v Rayonakh Sopryageniya Shelfa i Kontinentalnogo Sklona v Chernom More [Intensification of Vertical Turbulent Exchange in Areas of Pairing of the Shelf and the Continental Slope in the Black Sea]. In: NASU, 2008. Dopovidi Natsional'noyi Akademii Nauk Ukrayiny [Reports of the National Academy of Sciences of Ukraine], 6, pp. 108-112 (in Russian). 